\title{
Method to Detect Molecular Ranges in Elastic Lidar Signal
}

\section{Método para Detectar Rangos Moleculares en Señales Lidar Elásticas}

\author{
Pallotta, Juan V. ${ }^{1 *}$, Otero, Lidia A. ${ }^{1}$, Ristori, Pablo R. ${ }^{1}$, Quel, Eduardo J. ${ }^{1}$ \\ 1. CEILAP-UNIDEF (MINDEF-CONICET) UMI-IFAECI-CNRS 3351, Juan Bautista de la Salle \\ 4397 (B160ALO), Villa Martelli, Buenos Aires, Argentina. \\ ${ }^{*}$ E-mail: juanpallotta@gmail.com
}

Received: 19/10/2016 Accepted: 18/08/2017

DOI: $10.7149 /$ OPA.50.3.49015

\begin{abstract}
:
A method to detect aerosol plumes or clouds from an elastic lidar signal is presented, as well the determination of the atmospheric boundary layer height. It is based on the Rayleigh-fit concept, where the range-corrected elastic lidar signal is fitted with a pure-Rayleigh range-corrected lidar signal formed by radiosonde data. To run the algorithm, only temporal averaging has to be taken into account, and only one input parameter is needed. An analysis of the method is performed using real lidar data from different lidar system, showing the results and its limitations.
\end{abstract}

Key words: lidar; Rayleigh-fit; aerosol plume detection.

\section{RESUMEN:}

Se presenta un método para la detección de plumas de aerosoles o nubes en una señal lidar elástica, como también, la determinación de la altura de capa limite atmosférica. El objetivo de este trabajo es discriminar plumas de aerosoles o nubes de rangos Rayleigh puros, como también, la determinación de la altura de la capa limite atmosférica. Este método está basado en el concepto denominado Rayleigh-fit, donde la señal elástica corregida en rango es ajustada con una señal lidar Rayleigh pura formada con datos de radiosondeo. Para ejecutar este algoritmo, solo es necesaria de promediación temporal, y solo un parámetro de entrada es necesario para la ejecución del método. Se realiza un análisis del método con señales medidas de diferentes sistemas lidar y se muestran sus resultados y limitaciones.

Palabras clave: lidar; Rayleigh-fit; detección de pluma de aerosoles.

\section{REFERENCES AND LINKS / REFERENCIAS Y ENLACES}

[1] V. Freudenthaler. "Lidar Rayleigh-fit criteria," EARLINET-ASOS 7th Workshop (2009).

[2] Holger Baars, Thomas Kanitz, Ronny Engelmann, Dietrich Althausen, Birgit Heese, Mika Komppula, Jana Preißler, Matthias Tesche, Albert Ansmann, Ulla Wandinger, Jae-Hyun Lim, Joon Young Ahn, Iwona S. Stachlewska, Vassilis Amiridis, Eleni Marinou, Patric Seifert, Julian Hofer, Annett Skupin, Florian Schneider, Stephanie Bohlmann, Andreas Foth, Sebastian Bley, Anne Pfüller, Eleni Giannakaki, Heikki Lihavainen, Yrjö Viisanen, Rakesh Kumar Hooda, Sérgio Nepomuceno Pereira, Daniele Bortoli, Frank Wagner, Ina Mattis, Lucja Janicka, Krzysztof M. Markowicz, Peggy Achtert, Paulo Artaxo, Theotonio Pauliquevis, Rodrigo A. F. Souza, Ved Prakesh Sharma, Pieter Gideon van Zyl, Johan Paul Beukes, Junying Sun, Erich G. Rohwer, Ruru Deng, Rodanthi-Elisavet Mamouri and Felix Zamorano. "An overview of the first decade of PollyNET: an emerging network of automated Raman-polarization lidars for continuous aerosol profiling," Atmos. Chem. Phys., 16, 5111-5137 (2016). https://doi.org/10.5194/acp-16-5111-2016

[3] J. Bösenberg, V. Matthias, A. Amodeo, V. Amoiridis, A. Ansmann, J. M. Baldasano, I. Balin, D. Balis, C. Böckmann, A. Boselli, G. Carlsson, A. Chaikovsky, G. Chourdakis, A. Comerón, F. De Tomasi, R. Eixmann, V. Freudenthaler, H. Giehl, I. Grigorov, A. Hågård, M. Iarlori, A. Kirsche, G. Kolarov, L. 
Komguem, S. Kreipl, W. Kumpf, G. Larchev^eque, H. Linné, R. Matthey, I. Mattis, A. Mekler, I. Mironova, V. Mitev, L. Mona, D. Müller, S. Music, S. Nickovic, M. Pandolfi, A. Papayannis, G. Pappalardo, J. Pelon, C. Pérez, R. M. Perrone, R. Persson, D. P. Resendes, V. Rizi, F. Rocadenbosch,. A. Rodrigues, L. Sauvage, L. Schneidenbach, R. Schumacher, V. Shcherbakov, V. Simeonov, P. Sobolewski, N. Spinelli, I. Stachlewska, D. Stoyanov, T. Trickl, G. Tsaknakis, G. Vaughan, U. Wandinger, X. Wang, M.Wiegner, M. Zavrtanik, and C. Zerefos. "EARLINET: A European Aerosol Research Lidar Network to Establish an Aerosol Climatology," Max-Planck-Institut Report No. 348 (2003).

[4] Juan Pallotta, Pablo Ristori, Lidia Otero, Fernando Chouza, D’Elia Raul, Francisco Gonzalez, Alberto Etchegoyen, Eduardo Quel, en representación del consorcio Cherenkov Telescope Array (CTA). "Remote control and telescope auto-alignment system for multiangle LIDAR under development at CEILAP, Argentina," proceedings of the "Atmospheric Monitoring for High Energy Astroparticle Detectors" (AtmoHEAD), Saclay (France). arXiv:1309.6535 (2013).

[5] Juan Pallotta, Pablo Ristori, Lidia Otero, Fernando Chouza, D’Elia Raul, Francisco Gonzalez, Alberto Etchegoyen, Eduardo Quel, on behalf of Cherenkov Telescope Array (CTA). "Argentinian multiwavelength scanning Raman lidar to observe night sky atmospheric transmission," proceedings of the 33rd International Cosmic Ray Conference (ICRC), Rio de Janeiro, Brazil. arXiv:1307.5028 (2013).

[6] PhD thesis of Lidia Ana Otero. "Estudio de las propiedades ópticas de aerosoles en Argentina con técnicas de sensado pasivo y activo de la atmósfera," Universidad Nacional de Buenos Aires (2007).

[7] Vladimir Kovalev, William E. Eichinger, Elastic Lidar. Theory, Practice, and Analisys Methods, John Wiley \& Sons, Inc. (2004). https://doi.org/10.1002/0471643173

[8] Melfi, S. H., J. D. Spinhire, S. H. Chou, and S. P. Palm. "Lidar Observations of Vertically Organized Convection in the Planetary Boundary Layer over the Ocean," J. Clim. Appl. Meteor., 24, 806-821 (1985). https://doi.org/10.1175/1520-0450(1985)024<0806:LOOVOC>2.0.C0;2

[9] Shiv R. Pal, Wolfgang Steinbrecht, and Allan Carswell. "Automated method for lidar determination of cloud-base height and vertical extent," Appl. Opts., Vol. 31, 10 (1992).

[10] Massimo Del Guasta, Malice Morandi, Leopoldo Stefanutti. "One Year of Cloud Lidar Data From Dumont d'Urville (Antarctica). General Overview of Geometrical and Optical Properties," Journal Of Geophysical Research, Vol. 98, (1993).

https://doi.org/10.1029/93JD01476

[11] Cyrille Flamant, Jacques Pelon, Pierre H. Flamant, Pierre Durand. "Lidar Determination Of The Entrainment Zone Thickness At The Top Of The Unstable Marine Atmospheric Boundary Layer," Boundary-Layer Meteorology 83, 247-284, Kluwer Academic Publishers (1997).

[12] V. Matthias, D. Balis, J. Bosenberg, R. Eixmann, M. Iarlori, L. Komguem, I. Mattis, A. Papayannis, G. Pappalardo, M. R. Perrone and X. Wang. "Vertical aerosol distribution over Europe: Statistical analysis of Raman lidar data from 10 European Aerosol Research Lidar Network (EARLINET) stations," Journal of Geophysical Research, vol. 109, D18201 (2004). https://doi.org/10.1029/2004JD004638

[13] William P. Hopper and Edwin W. Eloranta. "Lidar Measurements of Wind in the Planetary Boundary Layer: The Method, Accuracy and Results from Joint Measurements with Radiosondeo and Kytoon," Journal of climate and applied meteorology, vol. 25, 990-1001 (1986). https://doi.org/10.1175/1520-0450(1986)025<0990:LMOWIT>2.0.C0;2

[14] Antti K. Piironen and Edwin W. Eloranta. "Convective boundary layer mean depths and cloud geometrical properties obtained from volume imaging lidar data. Journal of Geophysical Research," Vol. 100, 569-576 (1995). https://doi.org/10.1029/94JD02604

[15] Spinhirne, J. D., S. Chudamani, J. F. Cavanaugh, and J. L. Bufton. "Aerosol and Cloud Backscatter at 1.06, 1.54, and $0.53 \mathrm{Mm}$ by Airborne Hard-Target Calibrated Nd:YAG/methane Raman Lidar," Appl. Opt., 36, 3475-3490 (1997). https://doi.org/10.1364/A0.36.003475

[16] Laurent Menut, Cyrille Flamant, Jacques Pelon, and Pierre H. "Urban boundary-layer height determination from lidar measurements over the Paris area," Appl. Opt. 38, 945-954 (1999). https://doi.org/10.1364/A0.38.000945 
[17] Federico Angelini, Gian Paolo Gobbi. "Some remarks about lidar data preprocessing and different implementations of the gradient method for determining the aerosol layers," Annals Of Geophysics, 57, 2, A0218 (2014).

https://doi.org/10.4401/ag-6408

\section{Introduction}

A method to detect pure-Rayleigh ranges (and aerosols/clouds ranges) in lidar signals based on a Rayleigh-fit concept [1] [2] is presented. This procedure is used in EARLINET network [3] to check the quality of the signal in the far range and to determine the reference height for the normalization during the elastic inversion. In the Rayleigh-fit method, a pure-Rayleigh range-corrected lidar signal is fitted to a range-corrected elastic lidar signal over several ranges through an interval $\Delta r$ previously defined. Then, several tests are done in order to check if the range selected is molecular or not, and if not, a new range has to be chosen to repeat the procedure. The Rayleigh-fit criteria can be summarized in three steps as: 1 ) calculate Rayleigh fits for several $\Delta r, 2$ ) assess the determined $\Delta r$ and 3) find the optimum $\Delta r$ to be defined as pure-Rayleigh range. After the Rayleigh-fit has been calculated for as many reference height intervals, quality tests are applied to assess their validity, like analysis of the residuals, white noise criteria and signal-to-noise ratio (SNR) check [2]. In this work, the Rayleigh-fit is applied under the same concept: to discriminate pure-Rayleigh to the aerosols ranges in an elastic lidar signal. The difference lies in the criteria to define if the range analyzed can be computed as pure-Rayleigh or not.

The core of the method is to compute the Rayleigh-fit through all useful ranges of the acquired rangecorrected elastic lidar signal versus a pure-Rayleigh range-corrected lidar signal. The degree of similarity between both signals is quantified by the root-mean-square (RMS) error, and compared with the values obtained in other ranges of the lidar track.

For a successful result, signals must meet the following conditions:

- The lidar signal acquired must have both aerosols and molecular ranges in the interval of analysis. This means that there should be ranges of the free troposphere in the lidar track recorded.

- $\quad$ The lidar signal must be correctly aligned, following a pure-Rayleigh profile without any extra modulation.

- Signals with high level of random noise need to be temporally averaged. The number of averaged profiles depends of the aerosol load of the signal and analysis has to be made previous the application of the algorithm.

This method provides a solution to:

- $\quad$ Detect free troposphere ranges in the lidar signal, and therefore aerosol/clouds plumes.

- Find reference height (pure molecular range) for the normalization of the elastic lidar signal in the inversion procedure.

- Determine the atmospheric boundary layer (ABL) height as the first point of the first pureRayleigh range detected. No entrainment zone is identified.

A detailed approach of this method is described in next sections.

\section{Concept of the method}

The method works with measured elastics range-corrected lidar signals (Eq. 1) and a pure-Rayleigh range-corrected lidar signal (Eq. 2) calculated with radiosonde data. To obtain the range-corrected signals $\left(S^{e l}(r)\right.$ and $\left.S^{m}(r)\right)$, background noise bias has to be subtracted to the acquired data. Mathematically, both signals can be expressed as:

$$
\begin{gathered}
P^{e l}(r) r^{2}=S^{e l}(r)=K_{i}\left[\beta^{m}(r)+\beta^{p}(r)\right] e^{-2 \int_{0}^{r}\left[\alpha^{m}(r)+\alpha^{p}(r)\right] d r}+\delta P(r) r^{2} \\
P^{m}(r) r^{2}=S^{m}(r)=\beta^{m}(r) e^{-2 \int_{0}^{r} \alpha^{m}(r) d r}
\end{gathered}
$$

Where:

$P^{e l}(r)$ : Elastic lidar signal acquired.

$P^{m}(r)$ : Pure Rayleigh lidar signal. 
$r$ : Range.

$K_{i}$ : Instrumental constant.

$\beta^{x}(r)$ : Backscattering coefficient related to molecules and aerosol.

$\alpha^{x}(r)$ : Extinction coefficient related to molecules and aerosol.

$\delta P(r)$ : Random noise of the signal acquired.

The method is based on the quantification of the $S^{e l}(r)$ in terms of $S^{m}(r)$, in order to define the likeness between two signals. This evaluation is made by reducing the RMS error between both signals, where the lowest RMS error between $S^{e l}(r)$ and $S^{m}(r)$ defines the pure-Rayleigh range. An example of this concept can be seen in figure 1 , where in a free-aerosol range $\left(r_{m} \gtrsim 5 \mathrm{~km}\right), S^{m}(r)$ is affected by a factor " $\boldsymbol{a}$ " reducing the RMS error versus $S^{e l}(r)$.

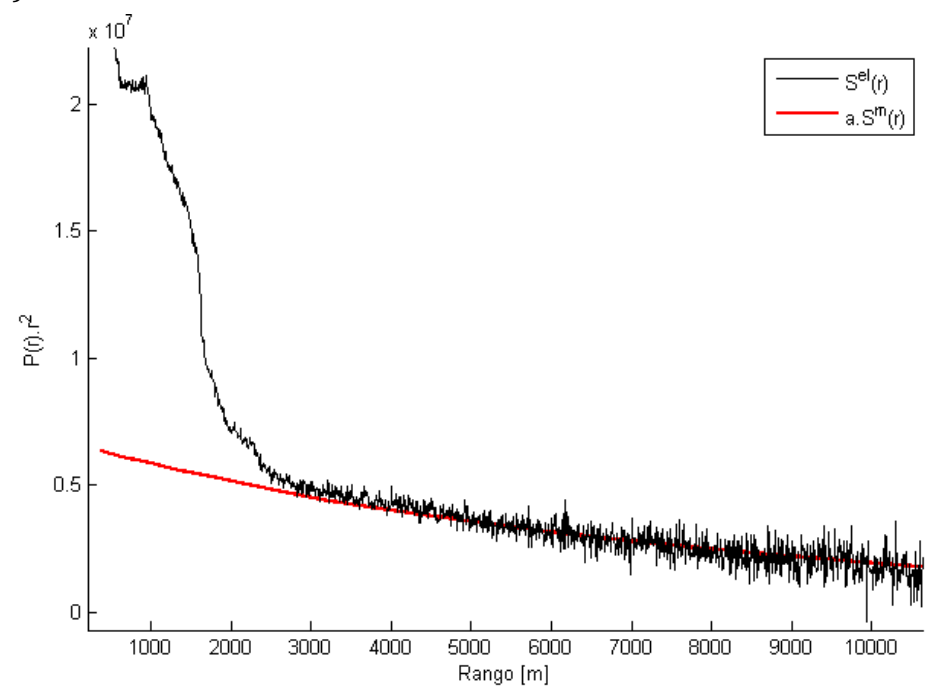

Fig. 1. Elastic lidar signal (black line) and pure molecular lidar signal (red line) fitted over a molecular range in order to minimize the RMS error.

In this example, no clouds or aerosol plumes are detected beyond the ABL. As can be seen, the lidar signal (black line) can be approximated in the free-aerosol range with a fitted pure-Rayleigh range-corrected lidar signal (red line). This can be seen mathematically reordering Eq. 1 over a pure molecular range $r_{m}$, where $\alpha_{p}\left(r_{m}\right)=\beta_{p}\left(r_{m}\right)=0$ :

$$
S^{e l}(r)=\left[K_{i} e^{-2 \int_{0}^{r m} \alpha^{p}(r) d r}\right] \beta^{m}(r) e^{-2 \int_{0}^{r} \alpha^{m}(r) d r}+\delta P(r) r^{2}
$$

Where in Eq. 3, the factor $\left[K_{i} e^{-2 \int_{0}^{r_{m}} \alpha^{p}(r) d r}\right]$ is applied to the pure-Rayleigh lidar signal (Eq. 2), plus the term related to range-corrected random noise $\left(\delta P(r) \cdot r^{2}\right)$. It is important to mention that this factor is constant over molecular ranges $\left(r_{m}\right)$, because in that region $\alpha^{p}\left(r_{m}\right)=0$ and its integral $\int_{0}^{r_{m}} \alpha^{p}(r) d r$ become constant and equal to the total aerosol optical depth (AOD) over previous ranges [0- $\left.r_{m}\right]$. The values of $K_{i}$ and $\alpha_{p}(r)$ are a-priori unknown, but its product $\left[K_{i} e^{-2 \int_{0}^{r_{m}} \alpha^{p}(r) d r}\right]$ can be estimated by fitting $S^{m}(r)$ to $S^{e l}(r)$ over molecular range minimizing the RMS error. In next section, a method to determine pure-Rayleigh ranges is presented.

\section{Method to detect pure-Rayleigh ranges}

In order to detect pure-Rayleigh ranges, an exploration windows $\Delta r$ is defined to perform a Rayleigh-fit throughout the all useful ranges previously defined $\left(\left[r_{\min }-r_{\max }\right]\right)$. A range $\left[r_{x} ;\left(r_{x}+\Delta r\right)\right]$ is defined as pureRayleigh if the RMS error of the Rayleigh-fit between $S^{m}(r)$ and $S^{e l}(r)$ is minimum. The fitting of both signals is performed by a simple linear correlation in the range where $\Delta r$ is placed $([r ;(r+\Delta r)])$ :

$$
\frac{d}{d a} \sum_{r}^{(r+\Delta r)}\left[S^{e l}(r)-a S^{m}(r)\right]^{2}=0
$$

Solving Eq. (4) for " $a$ ": 


$$
a(r ;(r+\Delta r))=\frac{\sum_{r}^{(r+\Delta r)}\left[S^{e l}(r) S^{m}(r)\right]}{\sum_{r}^{(r+\Delta r)}\left[S^{m}(r)\right]^{2}}
$$

And the RMS error in the range $[r ;(r+\Delta r)]$ is calculated with Eq. 6:

$$
R M \operatorname{Serr}(r ;(r+\Delta r))=\sqrt{\frac{1}{\Delta r} \sum_{r}^{r+\Delta r}\left[S^{e l}(r)-a S^{m}(r)\right]^{2}}
$$

Equations 5 and 6 are applied to each position $[r ;(r+\Delta r)]$ along the useful points of the lidar signal $\left(\left[r_{m_{i n}}{ }^{-}\right.\right.$ $\left.r_{\text {max }}\right]$ ), increasing $r$ in one bin till the end of the track. In each position of the exploration windows $\Delta r$, the parameters $\boldsymbol{a}$ and $\boldsymbol{R M S e r r}$ are computed, and $\boldsymbol{R M S e r r}$ is saved. Finally, the range $[r ;(r+\Delta r)]$ which has the minimum $\boldsymbol{R M S e r r}$ is defined as pure-Rayleigh or aerosol-free range. The procedure continues repeating the scan with the exploration windows $\Delta r$ starting forward higher altitudes from the last pure-Rayleigh range detected.

To clarify the method, a flowchart of the algorithm is shown in figure 2 .

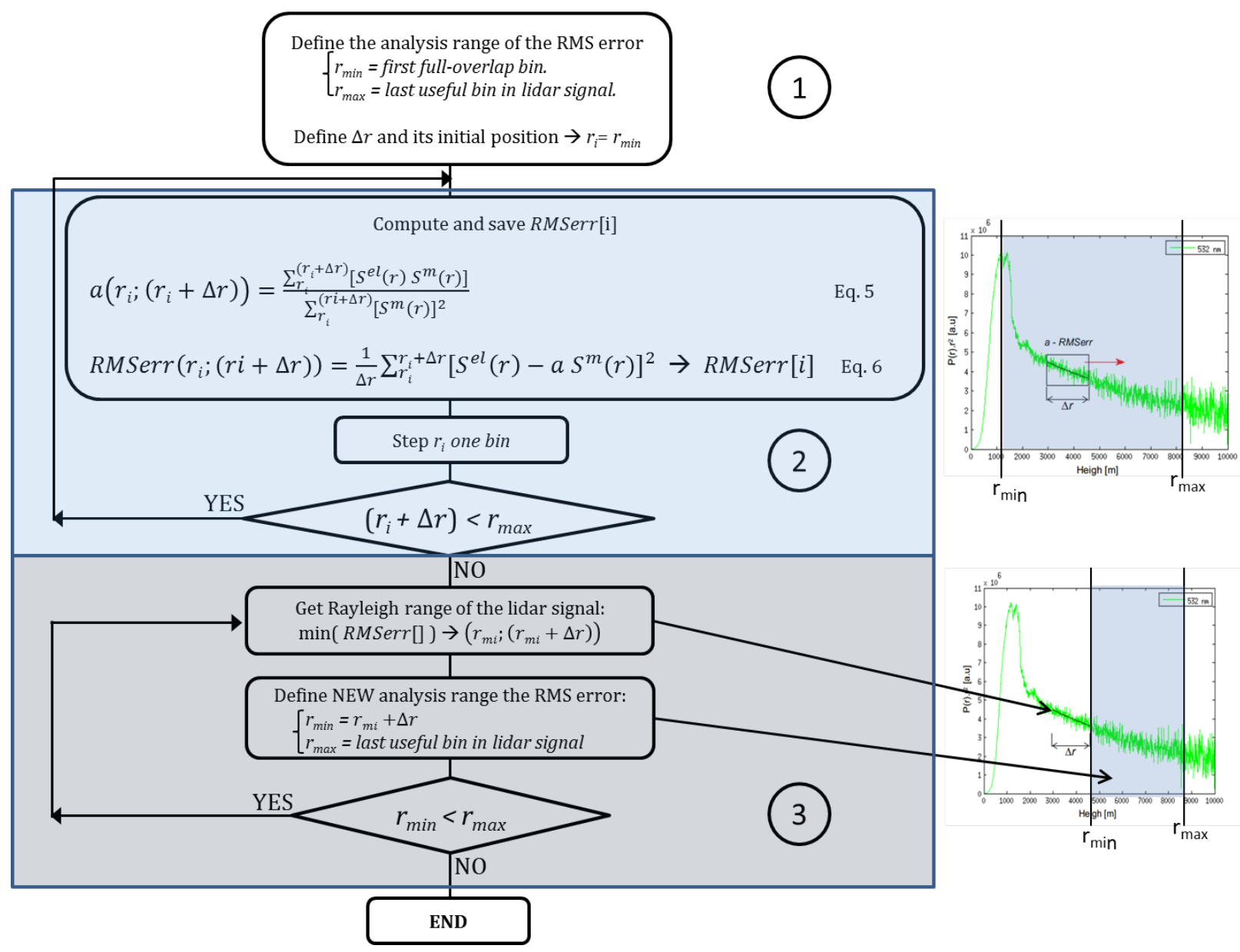

Figure 2. Flowchart of the algorithm to detect pure-Rayleigh ranges in a range-corrected elastic lidar signal.

In the first part of the flowchart (1), the inspection variables are established: width of $\Delta r$, its minimum and maximum ranges $\left[r_{\text {min }}-r_{\text {max }}\right]$ and its starting coordinate $r_{i}=r_{\text {min }}$.

In the second part of the flowchart (2), the fitting procedure of $S^{m}(r)$ to $S^{e l}(r)$ is made, and the RMS error is computed and saved in the variable RMSerr[i]. Then, $r_{i}$ is increased in one bin and, if $\left(r_{i}+\Delta r\right)<r_{\text {max }}$, parameters $\boldsymbol{a}$ and $\boldsymbol{R M S e r r}[\boldsymbol{i}]$ are computed for the new position. This continues till the condition $\left(r_{i}+\Delta r\right)<$ $r_{\max }$ became true, reaching to the end of the lidar signal.

Finally, in the third part of the flowchart (3), an analisys of the profile RMSerr is made to determine the pure-Rayleigh ranges. A range $[r ;(r+\Delta r)]$ is defined as a pure-Rayleigh where the RMSerr [i] is minimum, 
wich means that higher similarity with a pure-Rayleigh signal is found thruough the explored range. This range should be the lowest molecular range over the range $\left[r_{\min }-r_{\max }\right]$, and no pure-Rayleigh ranges should exist at lower altitudes in the range $\left[r_{\min }-r_{\max }\right]$. Then, once detected a pure-Rayleigh range in the interval $[r ;(r+\Delta r)], r_{\min }$ is upgraded to the next position forward the last Rayleigh-fit detected.

An example of the first loop showed in the second part of the flowchart is shown in figure 3, where can be seen the Rayleigh-fit in 4 different sampling ranges: one placed inside the atmospheric boundary layer (red line), and three more over pure-Rayleigh ranges (black line). The signal showed on figure 3 were taken with the multiangle Raman lidar [4] [5] on 12 May 2015.

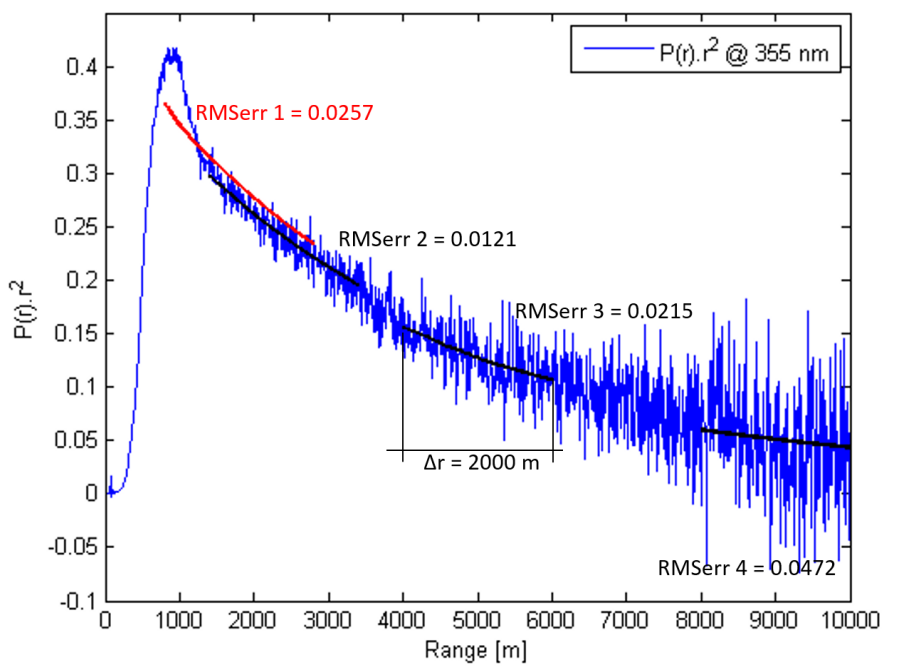

Figure 3. Pure-Rayleigh lidar signal (red and black lines) fitted over 3 different ranges in a range-corrected elastic lidar signal (blue line). Each of them are fitted to reduce the RMS error, showing its value next to the fit.

In the example showed in figure 3, the size of the exploration window $(\Delta r)$ was $2000 \mathrm{~m}$. As can be seen, the range chosen about 1300-3300 $\mathrm{m}$ has the lower RMS error of the four analyzed zones, therefore will be defined as a pure-Rayleigh. Here can be noticed that the method finds the lowest pure-Rayleigh range $\Delta r$ on the track $\left[r_{\min }-r_{\text {max }}\right]$ analyzed. The reason of this is due to the fact that the fit is performed over range-corrected signals, where the squared difference $\left[S^{e l}(r)-a S^{m}(r)\right]^{2}$ of equation 6 increase at higher altitudes due to the random noise is also range-corrected $\left(\delta P(r) . r^{2}\right)$. The method continues finding the minimum in the RMS error over the ranges beyond the last Rayleigh range detected (in the example of figure 3, forward $3300 \mathrm{~m}$ ).

\section{RMS error and its relations with the aerosol load and random noise}

A more detailed analysis of the RMS error shows the role of each parameter of the lidars equations. Replacing $S^{m}(r)$ and $S^{e l}(r)$ (Eqs. 1 and 2) into Eq. 6 and reordering conveniently can be obtained the Eq. 7.

$R M S e r r(r ;(r+\Delta r))=\sqrt{\frac{1}{\Delta r} \sum_{r}^{r+\Delta r}\left[\beta^{m}(r) T_{m}^{2}(r)\left[K_{i} T_{p}^{2}(r)-a\right]+K_{i} \beta^{p}(r) T_{m}^{2}(r) T_{p}^{2}(r)+\delta P(r) r^{2}\right]}$

Where:

$T_{m}(r)=e^{-2 \int_{0}^{r} \alpha^{m}(r) d r}$ : Molecular transmission from 0 to $r$.

$T_{p}(r)=e^{-2 \int_{0}^{r} \alpha^{p}(r) d r}$ : Aerosol transmission from 0 to $r$.

In last equation can be appreciated the role of the aerosol parameters $\beta^{p}(r)$ and $T_{p}^{2}(r)$ in the error formula. Equation 7 shows the RMS error in a range $[r ;(r+\Delta r)]$ (a particular step in the loop shown in part 2 of figure 3), its dependence to the aerosols conditions $\left(\beta_{p}(r)\right.$ and $\left.T_{p}(r)\right)$ and the range-corrected random noise $\left(\delta P(r) r^{2}\right)$. 
As stated before, the profile $R M S$ err r reach its lowest value when $\Delta r$ is placed in the lowest pure-Rayleigh range $\left(r_{m}\right)$ in the lidar track analyzed (block number (3) of the flowchart of the figure. 3 ). Under this condition:

- $\quad a=K_{i} T_{p}^{2}\left(r_{m}\right) \rightarrow$ due to $T_{p}^{2}(r m)=$ const over pure-Rayleigh range $\left(\alpha_{p}(r m)=0\right)$. See section 2 , Eq. 3 .

- $\beta^{p}\left(r_{m}\right)=0 \rightarrow$ due to $r_{m}$ is a pure-Rayleigh range.

And the Eq. 7 become only dependent to the range-corrected random noise $\left(\delta P(r) r^{2}\right)$ (Eq. 8).

$$
R M S \operatorname{Ser}(r ;(r+\Delta r))=\sqrt{\frac{1}{\Delta r} \sum_{r}^{(r+\Delta r)} \delta P(r) r^{2}}
$$

The capacity of the method to detect pure-Rayleigh ranges at lower altitudes is an advantage, and the first pure-Rayleigh height detected can be defined as the end of the ABL. In this sense, no detection of entrainment zone depth is made, since the method finds the altitude of the atmosphere that becomes aerosol-free without the transition between ABL and free troposphere.

The application of this method needs certain conditions related to the range-corrected random noise versus the range-corrected lidar signal intensity over aerosols/clouds ranges. In signals with poor temporal average, the $\boldsymbol{R M S e r r}$ over aerosol/cloud zones could be lower than pure-Rayleigh ranges, leading to wrong results. This means that aerosols/cloud range is more likely to be pure-Rayleigh than an aerosol/cloud range in terms of its RMS error.

An example of this scenario can be seen in figure 4, where the method is applied in signals with differents averaging time, and a cloud at $4 \mathrm{~km}$ approximately. In both cases, the algorithm is performed with $\Delta r=1000 \mathrm{~m}$, and the Rayleigh-fit and the RMS error profile is shown.

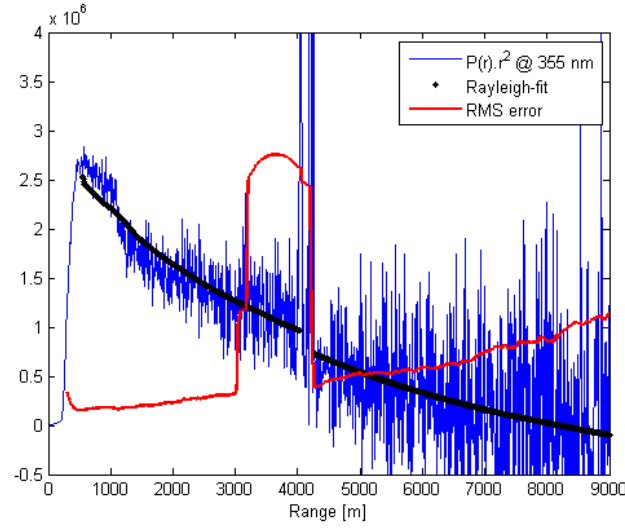

(a)

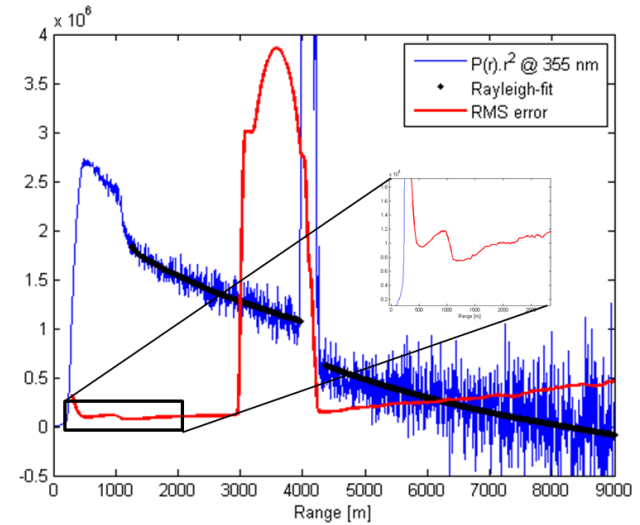

(b)

Figure. 4. $355 \mathrm{~nm}$ elastics lidar signals (blue line) from CEILAP lidar [6] at different average time: (a) $10 \mathrm{sec}$ and (b) $1 \mathrm{~min}$. In each plot, the Rayleigh-fit is shown (black line), using $\Delta r=1000 \mathrm{~m}$. The RMS error profile of the Rayleigh-fit is shown in red.

A wrong case can be seen in figure 4.a (blue line, 10 seconds acquisition time), where the Rayleigh-fit (black line) starts inside the ABL. This behavior is due to the $\boldsymbol{R M S e r r}$ profile (red line) in the ABL is lower than in pure-Rayleigh ranges at higher altitudes and high level of random noise.

In figure 4.b, the lidar signal (blue line) was obtained by averaging 6 profiles (1 minute of acquisition time), and the method works as expected. Here, the $\boldsymbol{R M S e r r}$ profile reaches its first minimum at the end of $\operatorname{ABL}\left(r_{m} \sim 1300 \mathrm{~m}\right)$, and then, the ranges between $r_{m}$ and $\left(r_{m}+\Delta r\right)$ are defined as the first pure-Rayleigh zones. The method continues the same procedure: finding the minimum in the $\boldsymbol{R M S e r r}$ profile (red line), but starting at the height $\left(r_{m}+\Delta r\right)$ towards the end of the lidar signal. It's important to remark how the $\boldsymbol{R M S e r r}$ profile starts to increase its values at a range $\Delta r$ before the cloud, meaning that the rangecorrected lidar signal starts to break of the pure-Rayleigh profile. This allows finding the next minimum in the RMS error profile after the cloud.

Hence, before the method is applied, a consideration about the averaging time has to be taken into account in order to reduce the random noise. Despite this, it's important to remark that even under the scenario of 
high random noise (figure 4.a), the method was able to detect the cloud ( $\sim \mathrm{km}$ ), performing the Rayleighfit automatically before and after the inhomogeneity. In the same lidar signal, the aerosols in the ABL weren't detected due to its low intensity compared with the noise at higher ranges. In this sense, there is no automatic rule to define the right averaging time to perform the algorithm, and has to be defined empirically for each set of data.

\section{False-positive of the method and constraints for the width of the exploration windows $\Delta r$}

A false-positive is defined when Rayleigh ranges are bypassed and are not detected by the algorithm. This could happen when the minimum value of $\boldsymbol{R M S e r r}$ is not obtained in the lowest pure-Rayleigh range, and it is found at higher altitudes. This omission is produced by an erroneous selection of the $\Delta r$ width. An example of this scenario can be seen in figure 5, where an elastic range-corrected lidar signal with an inhomogeneity at $\sim 4 \mathrm{~km}$ is shown (blue line) with the Rayleigh-fit (black line) and the RMS error (red line) profile using two different $\Delta r$.

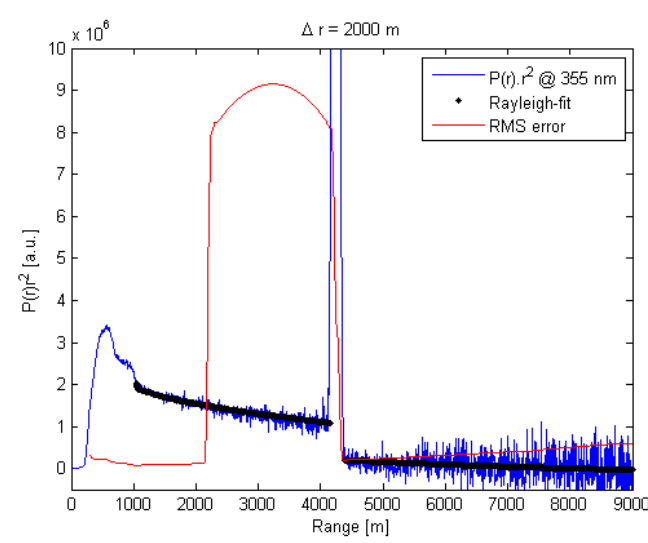

(a)

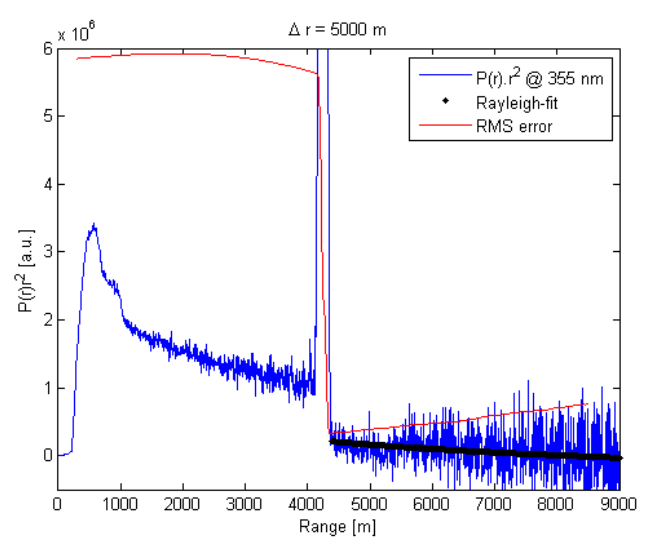

(b)

Figure. 5. A range-corrected lidar signal at $355 \mathrm{~nm}$ (blue line) with the result of the automatic Rayleigh-fit (black line) and its error profile (red line). (a) Well-selected $\Delta r(2000 \mathrm{~m})$, where the Rayleigh-fit is done across the all pure-Rayleigh ranges. (b) Erroneous $\Delta r$ width $(5000 \mathrm{~m})$ where Rayleigh range between ABL and the cloud is not detected. This is due to $\Delta r$ is higher than the gap between $\mathrm{ABL}$ and the cloud. Beyond the cloud, the fit is performed as espected.

In figure 5.a, a $\Delta r=2000 \mathrm{~m}$ is used, which is less than the gap between the two inhomogeneities (ABL and cloud), therefore, the pure-Rayleigh range between both inhomogeneities can be detected. In figure 4.b, the width of $\Delta r$ is larger than the gap in two aerosols zones, and the lower Rayleigh range is not detected by the algorithm. In this second case, the method starts to define molecular ranges after the cloud.

The $\boldsymbol{R M S e r r}$ profile (red line) shows its local minimum values at the beginning of each Rayleigh ranges detected. In figure 5.a, their local minimums are at the end of ABL and after the cloud. For the case showed in figure 5.b, its minimum it's at the end of the cloud, due to a large value of $\Delta r$, leaving the pure-Rayleigh ranges between $\mathrm{ABL}$ and the cloud undetected.

The value of $\Delta r$ is the only input of the method, being an easy parameter to define in situations where no aerosols plumes/clouds are below the planetary boundary layer, but require a more detalied inspection in a inhomogeneous atmosphere. In order to obtain an accurate value for $\Delta r$, two-dimensional images of the lidar backscatter signals are useful for this purpose. In this kind of plot (pcolor) is easy to visualize the whole data set, and can be used to estimate (temporal and spatial) clear zones, from others with aerosol/clouds. It is recommended a larger value of $\Delta r$ than lower ones, in order to have more information for computing the RMSerr, but this can lead to error described before. Narrow values are not recommended due to have few points to compute, leading pure-Rayleigh ranges undetected due to the random noise of the signal.

Figure 5.a and 4.b shows good results in the detection of pure-Rayleigh ranges by the evaluation of the range-corrected elastic lidar signal in terms of its similarity with a pure-Rayleigh lidar signal. This may be also used to detect the ABL heigh, defined as the first point of the first pure-Rayleigh range detected. Different data processing algorithms have been developed to discriminate the atmospheric layering from clear air. The general concepts of these methods are based on the detection of large levels of 
backscattering variance or gradient. The gradient methods find the height where the particle concentration decreases, whereas the variance methods find the height where particle scattering is the most variable [7].

One of the first methods to localize aerosols/clouds layers or ABL heights is based analyzing the spikes in the elastic lidar signal. In [8], the ABL height is determined where the backscatter intensity exceeds at least by $25 \%$ of more the intensity of the lidar signal from the free atmosphere. Later methods compute the derivative of the lidar signal profiles with respect to altitude. [9] and [10] reported the measurements of the cloud base height and vertical extent by analyzing the vertical derivative of the raw lidar signal. In [11], to determine the height of the ABL the first-derivative of the range-corrected lidar signal and the standard deviation profile is used, and the ABL limit is defined at which the standard deviation reach a threshold value. Another wide used method to detect the ABL height is [12], where the gradient is applied to the range-corrected elastic lidar signal. To avoid wrong results, low spatial resolution is used (30 m per bin) and a sliding-average filter of $240 \mathrm{~m}$ in order to compute the gradient without numerical problems. Methods like [13] and [14], deal with the variance in the lidar signal, evaluating this parameter through each altitude. The ranges who reach the local maximum are defined as the borders of ABL height, aerosols or clouds. This variance method performs well under convective regimes, being hard to use under stable layers. It's important to remark that, because of the large degree of variability of real atmospheric situations, the shape of real lidar signals and its random noise makes it difficult to implement these concepts with an automated method [7]. Also, the application of the derivative or the variance to a lidar signal depends on the spatial resolution of the range-corrected signals [12], or in other cases, the definition of threshold values (like in [8], [9] or [15]). Limitations of derivative and variance method have been reported in [16], where different metrological situations could lead to fail in the detection of ABL height.

The gradient method is the most used for ABL height detection, and much effort is done to make it more efficient. Also, it's important to remark that its results differ from a system to another, depending on the wavelength employed, and for systems having a low SNR, the direct differentiation of the range-corrected lidar signal may introduce unrealistic values, requiring spatial and temporal averages [17].

The method described in this work, no spatial averaging is a-priori needed, avoiding the chance to mask some features of the signal. Hence, temporal averaging is the only smoothing process to perform before the application of the algorithm.

\section{Case study}

In this section, two cases are analyzed using data sets from different lidars, both deployed at CEILAP laboratory. These two lidars have different hardware features, but the implementation of the method is the same: just the definition of the $\Delta r$ based on atmosphere condition, and a minimum of temporal averaging. The ABL height is compared with temperature profiles measured by nearest radiosounding at 9:30 AM local time, using the change of the temperature gradient profile to track the ABL height.

\section{6.a. Case study 1: Vertical lidar from CEILAP. Data set from 16 November, 2007}

The data used in this case were acquired by the multiwavelength Raman lidar system located at CEILAP (34.5으 S - 58.5 W, Villa Martelli, Buenos Aires, Argentina) [6]. This lidar uses a Continuum Surelite III $\mathrm{Nd}$ :YAG laser as emission system and the backscattered light is collected by a $0.4 \mathrm{~m}$ diameter Newtonian telescope. It contains a polychromator able to descriminate 3 elastics and 3 Raman wavelenths: 355, 532, $1064 \mathrm{~nm}$, the nitrogen lines for 355 and $532 \mathrm{~nm}$ (387 and $607 \mathrm{~nm}$ ), and water vapor (408 nm).

The pcolor of the $355 \mathrm{~nm}$ is showed in figure 6, where a spotted clouds between 3500 and $5000 \mathrm{~m}$ were detected before profile numbre 1800. The profiles were acquired with a spatial resolution of $6 \mathrm{~m}$, and temporal resolution of 10 seconds.

The $\Delta r$ can be defined by an inspection of the pcolor of the range-corrected lidar signal. For the case of the last figure, $\Delta r=2000 \mathrm{~m}$ (333 bins of $6 \mathrm{~m}$ each) was used. This value is enough to perform the Rayleigh-fit between inhomogeneities (ABL and clouds) across all data set.

In figure 7, four profiles are shown with the Rayleigh-fit in the pure-Rayleigh ranges automatically detected, showing a good correlation with the end of the entrainment zone and the borders of the cloud. This data set was acquired with a temporal resolution of $10 \mathrm{sec}$, but before applying the method, six 
profiles were averaged. This data set is the same used in figure 4, where for lower acquisition time, the method fails to detect the ABL height.

Also the temperature profile is shown, obtained from the nearest radiosonde $(\sim 30 \mathrm{~km}$ away from lidar site), taken at 9:30 AM. Good agreements can be appreciated between the first point were the Rayleigh-fit is made and the gradient inversion of the temperature profile.

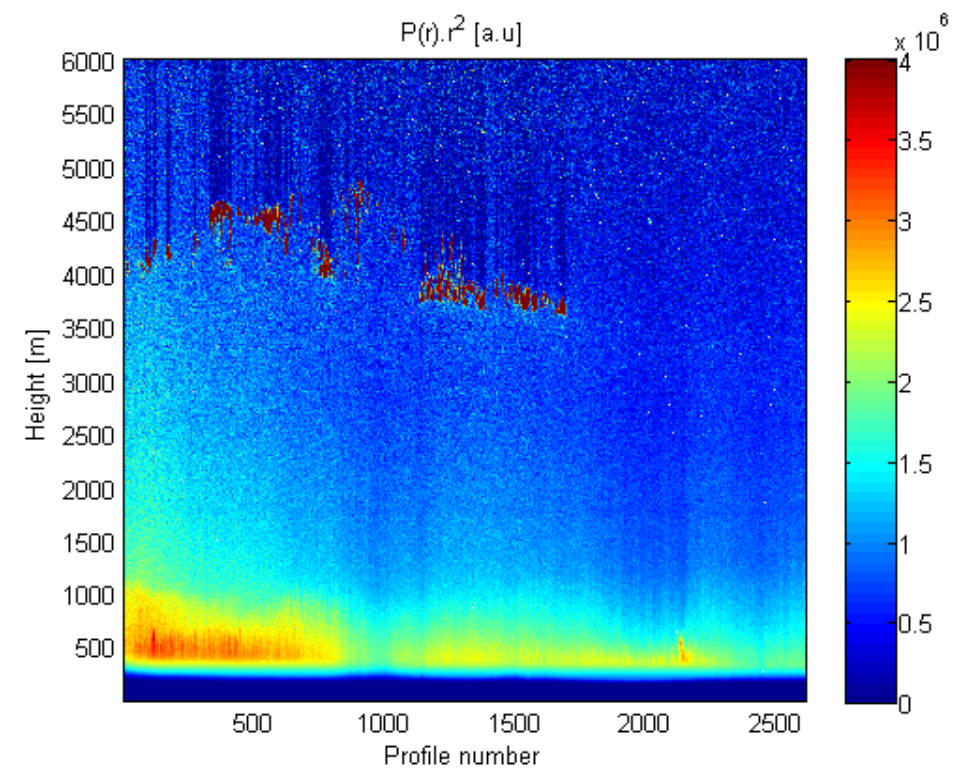

Figure 6. Pcolor of the lidar measurement from 16 Novenber of 2007 for $355 \mathrm{~nm}$. Data set start at 8:41 AM local time till 4:45 PM.
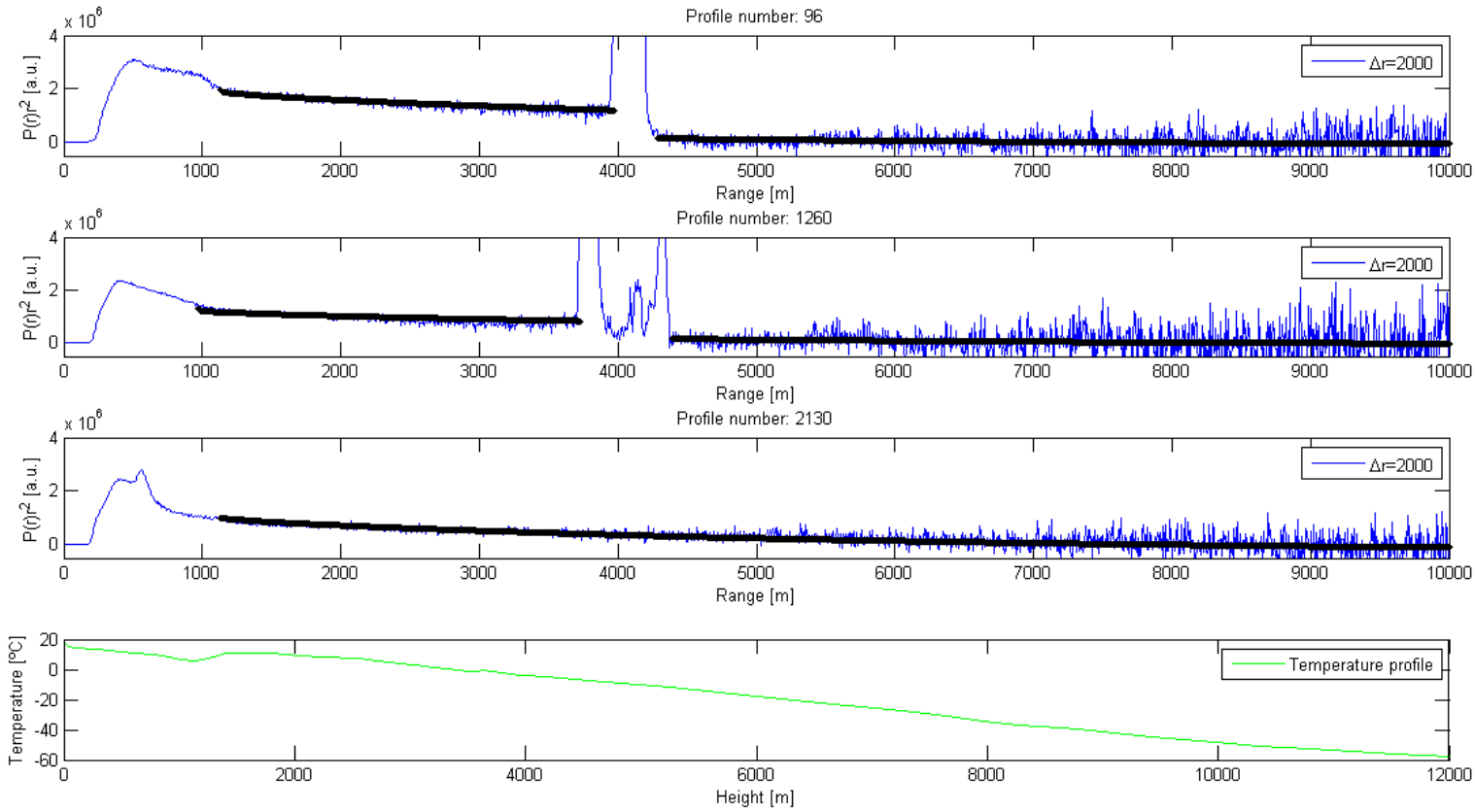

Figure 7. Tree lidar signals from de data set of the pcolor shown in figure 8 (profiles number 96, 1260 and 2130). In each plot is shown the lidar signal acquired (blue line) and the Rayleigh-fit (black line). In last sub-figure, the temperature profile is shown to track the gradient inversion to remark the ABL heigh.

\section{6.b. Case study 2: Multiangle Raman Lidar (MRL) from CEILAP. Data set from 24 June, 2015}

In this example, data from the multiangle Raman lidar of CEILAP [4] [5] is used. In this case, cirrus detection is shown, acquired with a spatial resolution of $7.5 \mathrm{~m}$ and temporal resolution of $10 \mathrm{sec}$. The pcolor is shown in figure 8.

This lidar has special features, like a big collection area formed by 6 Newtonian telescopes of $40 \mathrm{~cm}$ diameter each, making possible to acquire lidar data in low averaging time. In figure 9 , three profiles are 
shown (green line), with the results of the Rayleigh-fit applied automatically. A $\Delta r=2000 \mathrm{~m}$ is also used for this case.

In this case, $532 \mathrm{~nm}$ was used for the analysis, showing good agreement in the automatic application of the Rayleigh-fit across the pure-Rayleigh ranges. As a result of a large $\Delta r(2000 \mathrm{~m})$, undetected peak before the cirrus can be observed. Preferable large values of $\Delta r$ are recommended (when is possible) in order to work with a noisy signal, like used in last figure (10 sec acquisition time), where previous smoothing are need for other methods, like gradient or variance.

In these two cases, the method was applied over data sets from different lidars, with very different hardware and different wavelengths. Independently of the lidar system, the only input parameter needed is $\Delta r$, defined by the atmospherics conditions related to the minimum gap between two consecutives inhomogeneities. For each case, the temporal average needs are different due to have very different reception system: in case 2, 6 times higher reception area than case 1, made possible the detection of cirrus with only $10 \mathrm{sec}$ of acquisition time.

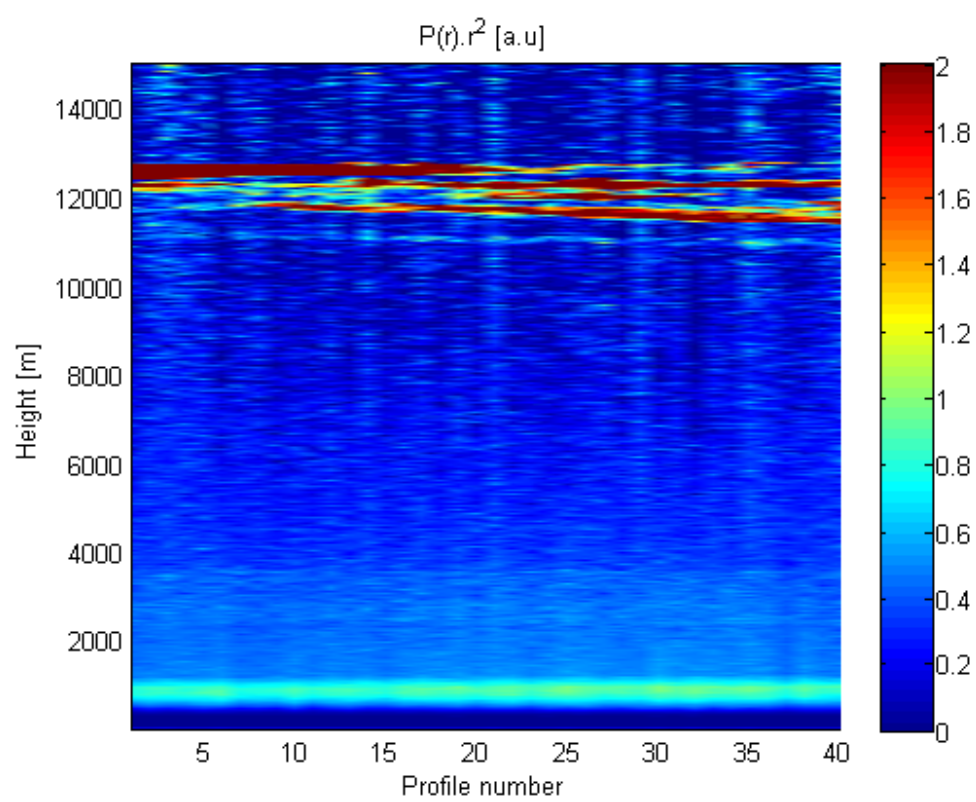

Figure 8. Pcolor for $532 \mathrm{~nm}$ of the multiangle Raman lidar, taken at 24 June 2015. Data set start at 7:30 PM local time, an only 40 profiles of $10 \mathrm{sec}$ acquisition time each is shown. The wavelenght shown is $532 \mathrm{~nm}$, for the profiles taken at zenithal direction.
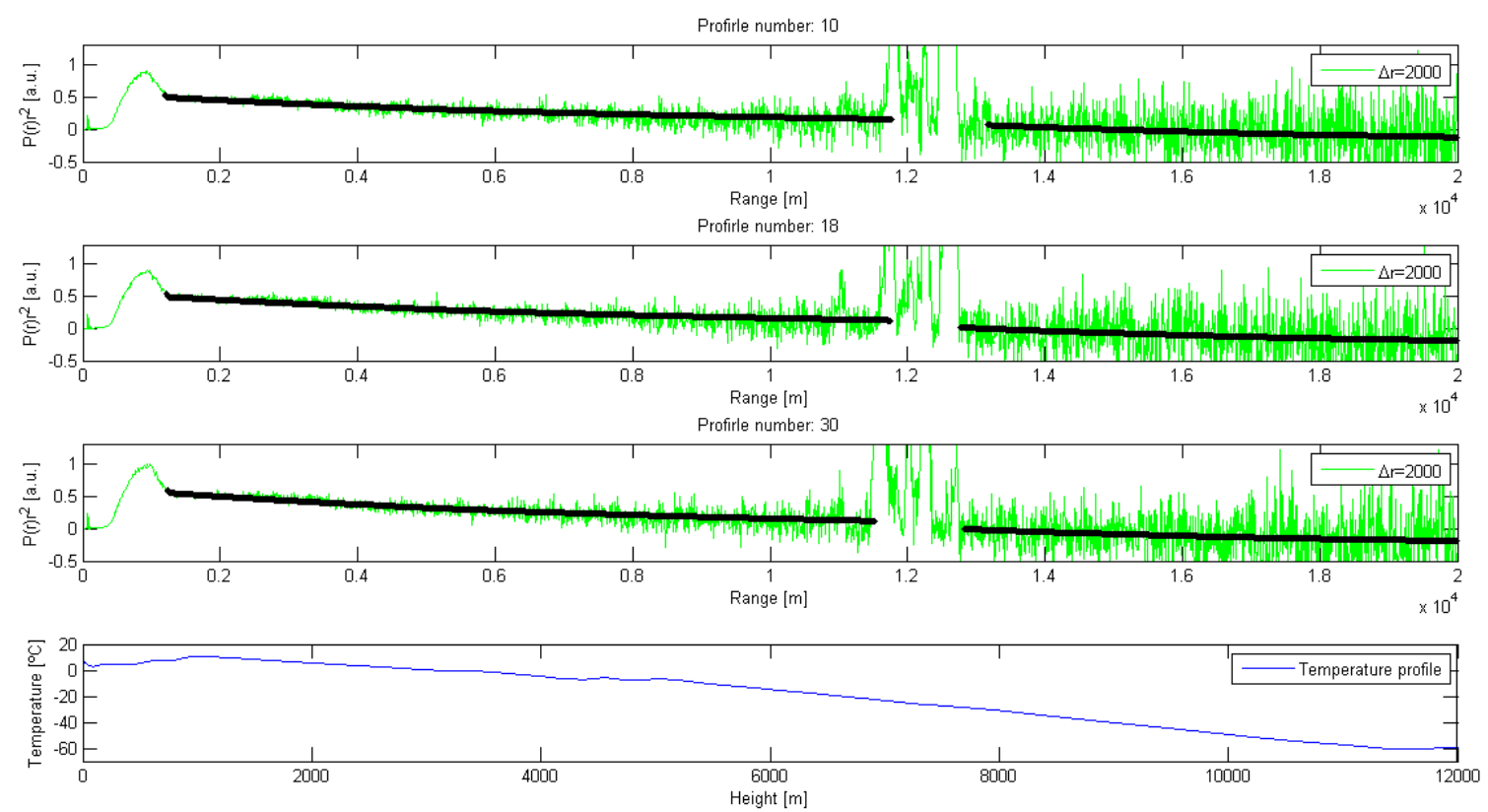

Figure 9. Tree lidar signals from data set of the pcolor shown in figure 8 (profiles number 12, 18 and 30). First 3 plots shown the $532 \mathrm{~nm}$ lidar signal acquired at $532 \mathrm{~nm}$ (green line) and the Rayleigh-fit (black line). In last sub-figure, the temperature profile 
taken at 9:30 AM is shown, where can be observed the gradient inversion to remark the ABL heigh.

\section{Summary}

A method to detect molecular ranges in an elastic lidar signal was presented. The algorithm is based on the Rayleigh-fit concept, which is applied across the pre-defined points of the lidar signal. The ranges of the lidar signal who compute the lower RMS error in the Rayleigh-fit are defined as a free-aerosols ranges. This method only need as an input the size of the exploration windows $\Delta r$, which has to be lower than the gap between two aerosols ranges. This parameter is related to the atmospheric conditions, and can be obtained by a visual inspection at the pcolor of the lidar data.

Temporal acquisition has to be taken into account to control the random noise of the signal, were very noisy signals could leads to erroneous results under certain scenarios. No spatial smoothing is a-priori needed; avoiding wrong averaging artifacts in the processed lidar signal.

The method was tested over real lidar data sets from different lidar and wavelengths. The results obtained shows good agreement when the ABL height is compared with temperature profiles.

Acknowledgments: The authors express their gratitude to Dr. Fernando Chouza who helps us to find the correct references, and Dr. Lucas Bali for the valuable discussion about certain topics of this paper.

This work were presented at the IX WLMLA (Workshop on Lidar Measurements in Latin America), held at Santos, Brazil from 17th to 22th July, 2016. 